\title{
What is the best treatment recommendation for HER2+ IBC with residual disease? - a narrative review
}

\author{
Danielle B. Zakon, Vicente Valero \\ Department of Breast Medical Oncology, The University of Texas MD Anderson Cancer Center, Houston, TX, USA \\ Contributions: (I) Conception and design: Both authors; (II) Administrative support: None; (III) Provision of study materials or patients: None; (IV) \\ Collection and assembly of data: Both authors; (V) Data analysis and interpretation: Both authors; (VI) Manuscript writing: Both authors; (VII) Final \\ approval of manuscript: Both authors. \\ Correspondence to: Vicente Valero, MD. Department of Breast Medical Oncology, Unit 1354, The University of Texas MD Anderson Cancer Center, \\ 1515 Holcombe Blvd., Houston, TX 77030, USA. Email: vvalero@mdanderson.org.
}

\begin{abstract}
Objective: The purpose of this article review is to discuss the current adjuvant approach for human epidermal growth factor receptor 2 (HER2) gene amplification or overexpression inflammatory breast cancer (IBC), as well as promising therapies to improve the prognosis of these patients, with the main focus on the high risk setting of patients with residual disease after neoadjuvant therapy.

Background: IBC is a rare and very aggressive form of breast cancer. HER $2+$ is more frequent in IBC than in non-IBC. A combined multimodality therapy is essential for better outcomes in non-metastatic HER2+ IBC patients, including neoadjuvant chemotherapy, with dual HER2 blockade, local therapy with surgery and radiotherapy, and adjuvant systemic therapy, which is defined on the basis of pathological response and hormone receptor (HR) status. Dual HER2 blockade with trastuzumab and pertuzumab combined to neoadjuvant chemotherapy improved the pathological complete response (pCR) rate. However, HER+ IBC patients with residual disease at surgery have a high risk of recurrence and death.

Methods: A comprehensive review was conducted through Medline/PubMed database, ClinicalTrials.gov, and conference abstracts.

Conclusions: Escalation of adjuvant therapy for patients with HER2+ IBC with residual invasive disease after neoadjuvant therapy is recommended. Adjuvant ado-trastuzumab emtansine and neratinib, for HR+ disease, are the most recent advances in this setting, with a clinically meaningful improvement in invasive disease-free survival (iDFS). Despite the clinical advances, reducing the risk of central nervous system recurrence remains an unmet need. Several promising clinical trials are ongoing to improve patients' prognosis in this high-risk of recurrence setting.
\end{abstract}

Keywords: Inflammatory breast cancer (IBC); human epidermal growth factor receptor 2 (HER2); residual disease

Submitted Oct 21, 2021. Accepted for publication Dec 21, 2021.

doi: $10.21037 / \mathrm{cco}-21-122$

View this article at: https://dx.doi.org/10.21037/cco-21-122

\section{Introduction}

Inflammatory breast cancer (IBC) is rare and is one of the most aggressive forms of breast cancer, with high rates of loco-regional and distant recurrence and mortality rates, despite advances in clinical treatment in recent decades. Due to the rarity of IBC and lack of prospective data, most of the systemic treatment recommendations are extrapolated from patients with locally advanced non-IBC and is tailored according to molecular subtype. Although IBC can be of any subtype, a higher prevalence of human epidermal growth factor receptor 2 (HER2) gene amplification or overexpression (HER2+) is found in IBC patients $(40 \%)$ than in non-IBC patients $(20 \%)(1,2)$. 
The standard of care for HER2+ IBC is a combined multimodality approach, that includes neoadjuvant therapy with dual anti-HER2 antibodies and anthracyclinebased chemotherapy with AC-THP (doxorubicin and cyclophosphamide, followed by paclitaxel or docetaxel with trastuzumab and pertuzumab) or non-anthracycline based chemotherapy with TCHP (docetaxel or paclitaxel, carboplatin, trastuzumab, and pertuzumab), followed by surgery, radiotherapy, and adjuvant systemic therapy (3-8). Although the optimal neoadjuvant chemotherapy backbone with dual HER2 blockade for IBC patients is not well-established, regimens that omit anthracyclines, such as TCHP, have been used progressively more often on the basis of similar pathological complete response (pCR) and event-free survival rates; these regimens are associated with lower rates of cardiac toxicity and a lower risk of developing acute myeloid leukemia and myelodysplastic syndrome (5,7-9).

Adjuvant systemic targeted therapy is defined on the basis of pathological response and hormone receptor (HR) status. A pCR, defined as the absence of residual invasive disease in the breast and lymph nodes (ypT0/Tis ypN0), is an early surrogate for long-term survival outcomes, principally in patients with high-proliferating tumors, such as in HER2+ breast cancer (10). Despite significant advances in neoadjuvant therapy for HER2+ breast cancer, around $40-55 \%$ of patients will not achieve a pCR. These patients are at substantial risk of distant relapse and death $(11,12)$. Therefore, new therapeutic strategies are clearly needed.

This review will focus on the current evidence for adjuvant therapy of HER+ IBC with residual disease and future perspectives to improve patients' prognosis in this high-risk of recurrence setting. We present the following article in accordance with the Narrative Review reporting checklist (available at https://dx.doi.org/10.21037/cco-21-122).

\section{Methods}

A literature review was conducted through Medline/ PubMed database and conference abstracts from American Society of Clinical Oncology, European Society for Medical Oncology, and San Antonio Breast Cancer Symposium. Only studies published in English between 2000 and November 2021 were included. The ClinicalTrials.gov database was also searched in November 2021 to identify ongoing clinical trials with inclusion of HER+ breast cancer patients with residual disease at surgery after neoadjuvant chemotherapy and HER2-directed therapy.

\section{Discussion}

\section{Adjuvant therapy for HER+ IBC patients with residual disease}

Ado-trastuzumab emtansine (T-DM1) is an antibody-drug conjugate with trastuzumab that is linked to derivative of maytansine 1 (DM1), a cytotoxic agent with potent inhibitory activity on microtubule polymerization. EMILIA was a pivotal phase III trial, that led to the approval of T-DM1 for patients with HER2+ advanced breast cancer, who had been previously treated with trastuzumab and taxane. T-DM1 resulted in superior progression-free survival (PFS) and overall survival (OS) duration compared to lapatinib plus capecitabine, even with $27 \%$ crossover from control to T-DM1 (13). The TH3RESA trial reaffirmed the role of T-DM1 in patients with metastatic disease, demonstrating a benefit of T-DM1 versus physician's choice treatment after disease progression on two or more HER2-targeted therapies (14).

Patients with HER2 + early breast cancer who do not achieve a pCR after neoadjuvant systemic therapy have a high risk of recurrence and death; this, along with the results of T-DM1 in trastuzumab-resistant HER2+ metastatic breast cancer, were the rationale for launch the KATHERINE trial, a phase III trial of 1,486 patients with HER2 + early breast cancer (77 with IBC), with breast or lymph node-invasive residual disease after neoadjuvant therapy with chemotherapy and HER2-targeted therapy. The majority of patients received anthracycline-based chemotherapy (76.9\%). All patients received taxane and HER2-targeted therapy with trastuzumab. However, only $19.5 \%$ of patients received a second anti-HER 2 therapy in addition to trastuzumab. Patients were randomly assigned to receive adjuvant therapy with T-DM1 or trastuzumab for 14 cycles (15).

Adjuvant T-DM1 was superior to trastuzumab, resulting in a $50 \%$ reduction in the risk of recurrence of invasive disease or death in 3 years $(12.2 \%$ versus $22.2 \%$; $95 \%$ CI: $0.39-0.64 ; \mathrm{P}<0.001)$. The benefit of T-DM1 over trastuzumab was relatively uniform across all subgroups, including in patients with minimal residual disease (ypT1a, ypT1mic, and N0) and regardless of the type of neoadjuvant chemotherapy, anthracycline or non-anthracycline-based $(15,16)$. Therefore, the KATHERINE trial established T-DM1 as the standard adjuvant therapy for patients with 
invasive residual disease at the time of surgery. Longer term follow-up data are required to evaluate the effect of adjuvant T-DM1 on OS duration. In general, T-DM1 was reasonably well tolerated, with minimal impact on patientreported quality of life. Nonetheless, as expected, T-DM1 had a higher toxicity rate than did trastuzumab, with a more pronounced incidence of thrombocytopenia (28.5\% versus $2.4 \%)$, particularly in patients who had previously received platinum therapy; peripheral neuropathy $(32.3 \%$ versus $16.9 \%)$, mainly in patients with baseline neuropathy; and pulmonary toxicity $(3.4 \%$ versus $1.0 \%)(16,17)$.

Conversion from HER2 + disease to HER2- disease in surgical specimens was evaluated in the KATHERINE trial. Changes occurred in $8.3 \%$ of the tested patients $(n=70)$. Interestingly, no invasive disease-free survival (iDFS) events were found among patients who were randomly assigned to T-DM1 $(\mathrm{n}=28)$, in contrast to 11 events in patients who were randomly assigned to trastuzumab (18). Although the sample size was small, the results of this exploratory analysis suggest that loss of HER2 expression after neoadjuvant therapy should not affect the indication for adjuvant T-DM1.

In summary, the impressive iDFS improvement from the KATHERINE trial with adjuvant T-DM1 in the high-risk population with invasive residual disease after neoadjuvant treatment, including IBC, highlights the need of the optimal adjuvant systemic therapy in the multimodality treatment of HER2 + non-metastatic IBC patients.

\section{Extended adjuvant therapy for HER+ IBC patients}

Neratinib, an irreversible pan-HER tyrosine kinase inhibitor, was evaluated in the phase III ExteNET trial as extended adjuvant therapy for 1 year versus placebo after neoadjuvant or adjuvant trastuzumab-based therapy. The study was positive for the primary endpoint of iDFS, A subgroup analysis of the population with $\mathrm{HR}+$ disease demonstrated an absolute benefit of $5.1 \%$ in 5 years (HR, 0.58 ; $95 \%$ CI: $0.41-0.82 ; \mathrm{P}=0.002)$. Among the patients with node-positive disease, which corresponded to $76 \%$ of the total population, a greater benefit was observed, principally in those with 4 or more involved lymph nodes $(19,20)$.

Furthermore, patients with HR+ residual disease showed a trend of benefit with neratinib, with a 5 -year iDFS rate of 7.4\% (HR 0.60, 95\% CI: 0.33-1.07; P=0.086) and a statistically and clinically meaningful OS benefit, with an absolute benefit of $9.1 \%$ at 8 years (HR, $0.47 \%$; $95 \%$ CI: 0.23-0.92; $\mathrm{P}=0.031)(21)$. This effect in $\mathrm{HR}+$ patients may be related to bidirectional cross-talk between the HER2 and estrogen receptors pathways, a known mechanism of resistance in HER2+/HR+ tumors (21).

The main toxicity of neratinib therapy was grade 3 diarrhea ( $40 \%$ of patients); most of the episodes occurred in the first month, leading to treatment discontinuation in $17 \%$ of patients. However, the CONTROL trial showed that this side effect can be significantly reduced with preemptive prophylaxis or progressive dose escalation (22).

The potential benefit of extended adjuvant therapy with neratinib after neoadjuvant or adjuvant pertuzumab or adjuvant T-DM1 is unknown. Nevertheless, considering the high risk of recurrence in patients with HER2 + IBC without $\mathrm{pCR}$, neratinib should be considered in patients with $\mathrm{HR}+$ disease.

\section{Adjuvant therapy for HER+IBC patients with pCR}

Although pCR is associated with favorable prognosis as compared to patients with residual disease, the initial clinical stage must be considered. Patients with locally advanced disease at diagnosis, such as IBC, continue to have a substantial risk of recurrence (15-20\%), despite a pCR $(4,23,24)$. Identifying biomarkers that can predict recurrence risk despite pCR in IBC is needed to determine which patients require treatment escalation to improve survival outcomes.

The APHINITY trial showed that the addition of pertuzumab to trastuzumab plus chemotherapy as adjuvant therapy, was beneficial in the node-positive cohort, regardless of HR status. In these patients, the addition of pertuzumab improved the iDFS rate at 6 years $(87.9 \%$ versus $83.4 \%$; HR, 0.72 ; $95 \%$ CI: $0.59-0.87$ ), mainly by a reduction in distant relapses (25). On the basis of these results and considering the remaining significant risk of recurrence in patients with IBC with pCR, continued trastuzumab and pertuzumab in the adjuvant setting is indicated until 1 year of dual HER2 blockade has been completed. Figure 1 summarize the adjuvant systemic treatment recommendations for HER2+ IBC.

\section{Future perspectives}

Recently, novel HER2-targeted agents were found to improve the treatment of patients with HER2 + metastatic breast cancer and may be effective in the adjuvant setting. Fam-trastuzumab deruxtecan-nxki (T-DXd), an antibodydrug conjugate that is composed of trastuzumab and a 


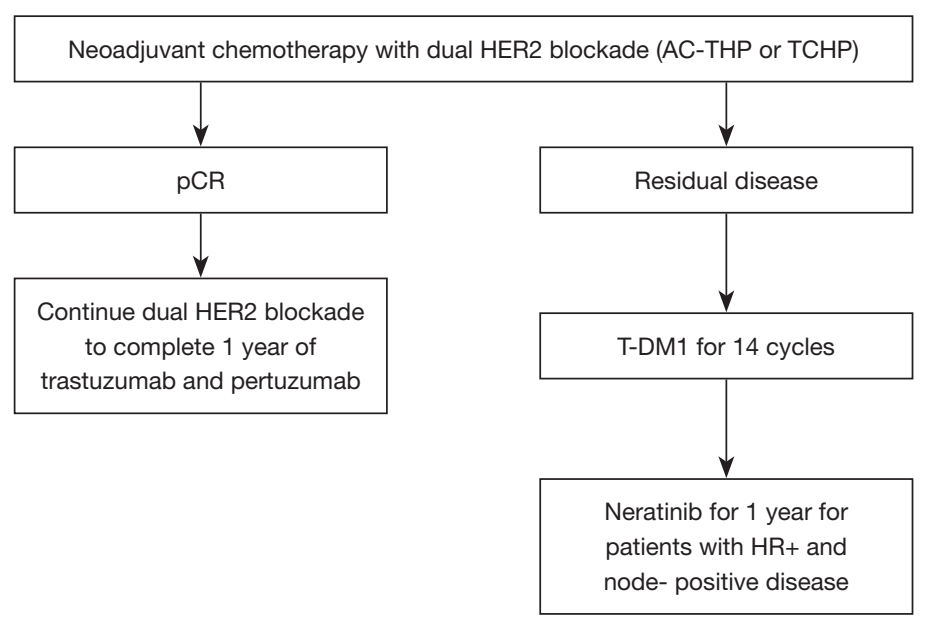

Figure 1 Proposed systemic treatment algorithm for patients with non-metastatic HER2+ IBC. HER2, human epidermal growth factor receptor 2 gene; IBC, inflammatory breast cancer; AC-THP, doxorubicin and cyclophosphamide, followed by paclitaxel or docetaxel with trastuzumab and pertuzumab; TCHP, docetaxel or paclitaxel, carboplatin, trastuzumab, and pertuzumab; pCR, pathological complete response; T-DM1, ado-trastuzumab emtansine; $\mathrm{HR}+$, hormone receptor-positive.

topoisomerase I inhibitor payload, was granted accelerated approval by the Food and Drug Administration in 2019, on the basis of the results of a phase II trial in patients who had received at least two prior anti-HER2 therapies. In the study, T-DXd demonstrated a high antitumor activity, with a response rate of $60.9 \%$ and PFS duration of 16.4 months (26). Subsequently, the phase III DESTINY-Breast03 trial showed a remarkable advantage in PFS and response rate of T-DXd versus T-DM1 in metastatic HER2+ breast cancer patients previously treated with trastuzumab and a taxane (27). In this context, T-DXd is being evaluated in the adjuvant setting versus T-DM1 in HER+ breast cancer patients with residual disease at surgery in the DESTINY-Breast05 trial (ClinicalTrials.gov identifier: NCT04622319).

Tucatinib is a novel tyrosine kinase inhibitor of HER2 that was approved by the Food and Drug Administration in 2020, on the basis of the results of the phase III HER2CLIMB trial, for patients who have received at least one prior antiHER2 therapy. The addition of tucatinib to trastuzumab and capecitabine resulted in prolonged PFS and OS durations in this population (28). Moreover, among the 291 patients with brain metastases at baseline included in the study, the tucatinib was associated with statistically significant improved intracranial response $(47.3 \%$ versus $20 \%$ ), CNS-PFS (9.9 versus 4.2 months), and OS (18.1 versus 12.0 months) (29).

CompassHER2 RD (ClinicalTrials.gov identifier: NCT04457596) is a promising ongoing trial with the aim of evaluating the potential benefit of adding tucatinib to T-DM1 as adjuvant therapy for patients with residual disease after neoadjuvant systemic therapy, including chemotherapy and anti-HER2 therapy. This trial is essential considering the need to reduce the risk of CNS recurrence in patients with HER2 + IBC with residual disease at surgery, the efficacious penetration of tucatinib into the $\mathrm{CNS}$, and the demonstrated activity of tucatinib in brain metastases.

The combination of immune checkpoint inhibitors to chemotherapy was a major breakthrough in the treatment of advanced and early stage triple negative breast cancer (30-32). For HER2 + metastatic breast cancer, the phase $1 \mathrm{~b} / 2$ PANACEA trial showed the preliminary efficacy of pembrolizumab, an anti-programmed death 1 monoclonal antibody, associated with trastuzumab in patients with trastuzumab-resistant disease. The antitumor activity was restricted to the cohort with expression of programmed death ligand 1 (PD-L1), with an objective response of $15 \%$ and median duration of disease control of 11.1 months in this group (33). Likewise, the phase II KATE2 study showed a trend of benefit in the PFS and OS duration with the addition of atezolizumab, an anti-PD-L1 monoclonal antibody, to T-DM1 in PD-L1 positive patients (34). Currently, the combination of atezolizumab with T-DM1 is being tested in the phase III ASTEFANIA trial as adjuvant therapy for HER2+ breast cancer patients with residual invasive disease post-neoadjuvant therapy (ClinicalTrials. gov identifier: NCT04873362). 
Table 1 Ongoing phase III studies with inclusion of HER2 + early breast cancer patients with residual invasive disease following neoadjuvant therapy

\begin{tabular}{llll}
\hline Study (ClinicalTrials.gov identifier) & Experimental arm & Control arm & Eligibility \\
\hline DESTINY-Breast05 (NCT04622319) & T-DXd $\times 14$ cycles & T-DM1 $\times 14$ cycles & $\begin{array}{l}\text { High risk HER2+ BC with RD } \\
\text { after NAT }\end{array}$ \\
CompassHER2 RD (NCT04457596) & T-DM1 + tucatinib $\times 14$ cycles & T-DM1 + placebo $\times 14$ cycles & $\begin{array}{l}\text { High risk HER+ BC with RD } \\
\text { after NAT }\end{array}$ \\
ASTEFANIA (NCT04873362) & T-DM1 + atezolizumab $\times 14$ cycles & T-DM1 + placebo $\times 14$ cycles & $\begin{array}{l}\text { High risk HER+ BC with RD } \\
\text { after NAT }{ }^{3}\end{array}$ \\
eMonarcHER (NCT04752332) & ET + abemaciclib $\times 26$ cycles $^{4}$ & ET + placebo $\times 26$ cycles & High risk HR+/HER2+ BC $^{5}$ \\
\hline
\end{tabular}

\begin{abstract}
${ }^{1}$, high risk breast cancer was defined as inoperable breast cancer at presentation (cT4, N0-3, M0 or cT1-3, N2-3, M0) with RD; or operable at presentation (T1-3, N0-1, M0) with pathological positive ALN (ypN1-3) after neoadjuvant therapy. ${ }^{2}$, high risk breast cancer was defined as cT1-4, cN0-3 disease at presentation and RD. Patients with cT1a/bN0 tumors at presentation are not eligible. cN0 eligible if $\mathrm{T}$ size $\geq 2.0 \mathrm{~cm}$. cN1-2 eligible if $\mathrm{T}$ size $\geq 1.5 \mathrm{~cm}$. $^{3}$, high risk breast cancer was defined as cT4/anyN/M0, any cT/N2-3/M0, or cT1-3/N0-1/M0, with RD. Patients with clinical stage $\mathrm{cT} 1 \mathrm{mi} / \mathrm{T} 1 \mathrm{a} / \mathrm{T} 1 \mathrm{~b} / \mathrm{N} 0$ at disease presentation are not eligible. Those with cT1-3/N0-1 disease must have pathological evidence of RD in ALN at surgery. ${ }^{4}$, after completing adjuvant HER2-targeted therapy. Physician's choice endocrine therapy. ${ }^{5}$, high risk breast cancer was defined depending on the initial therapy. If neoadjuvant therapy: RD in ALN (ypN+); if no NAT (surgery upfront): $\geq 4$ pathological positive ALN, or 1-3 pathological positive ALN and HG3 and/or T size $\geq 5 \mathrm{~cm}$. T-DXd, fam-trastuzumabderuxtecan-nxki; T-DM1, ado-trastuzumab emtansine; BC, breast cancer; RD, residual invasive disease; NAT, neoadjuvant therapy; HR+, hormone receptor-positive; ET, endocrine therapy; ALN, axillary lymph-node; HG3, histologic grade 3.
\end{abstract}

Patients with HR+/HER2+ IBC have lower pCR rates than patients with HR-/HER+ IBC. However, the effect of pCR status on survival outcome is not as pronounce as in patients with HR-/HER+ disease. Despite an overall better prognosis, patients with $\mathrm{HR}+\mathrm{HER}+\mathrm{IBC}$ have a higher rate of late recurrence than patients with HR-/ HER2+ IBC (11). During the past decade, the cyclindependent kinase 4 and 6 (CDK4/6) inhibitors significantly improved the treatment landscape for patients with $\mathrm{HR}+$ / HER2- advanced breast cancer. Recently, abemaciclib was the first CDK4/6 inhibitor approved in the adjuvant setting in combination with endocrine therapy for patients with HR+/HER2-, node-positive, high risk early breast cancer. Adjuvant treatment with abemaciclib led to improvement in iDFS (HR, 0.70; 95\% CI: 0.59-0.82) (35). Given the results of CDK4/6 inhibitors for HR+/HER2- breast cancer and the positive results showed in phase I and II trials for $\mathrm{HR}+$ / HER+ advanced breast cancer (36,37), the MonarcHER trial is going to assess the potential benefit of adding abemaciclib to standard adjuvant endocrine therapy for patients with HR+/HER+, who had completed adjuvant HER2-targeted therapy (ClinicalTrials.gov identifier: NCT04752332). The ongoing trials are summarized in Table 1 .

\section{Adjuvant bormone therapy and radiotherapy}

The optimal adjuvant endocrine therapy for IBC patients with $\mathrm{HR}+$ residual disease is essential, including ovarian suppression and an aromatase inhibitor for premenopausal patients (38) and an aromatase inhibitor for postmenopausal patients (39). As these patients are at substantial risk for late recurrence, extending adjuvant endocrine therapy for up to 10 years is strongly recommended $(39,40)$. Bone-targeted therapy is also recommended in postmenopausal patients with high-risk breast cancer, in addition to in premenopausal patients in whom menopause is induced by ovarian suppression, to reduce bone recurrence, improve survival, and decrease bone loss and related complications $(41,42)$.

Finally, the multimodality treatment for patients with stage III IBC includes adjuvant radiotherapy; which can be performed concomitantly with HER2-targeted adjuvant therapy, including T-DM1, according to the protocol of the KATHERINE trial (15), or with dual HER2 blockade with trastuzumab and pertuzumab (26).

\section{Conclusions}

The pCR is an important prognostic factor for HER2+ breast cancer. Patients with HER2 + IBC with residual invasive disease at surgery after neoadjuvant chemotherapy with HER2-targeted therapy have a high risk of recurrence and death; thus, escalation of adjuvant therapy is necessary to improve prognosis. Adjuvant T-DM1 is the most effective targeted therapy in this setting, with a significant 
reduction in invasive disease recurrence and an acceptable safety profile. Extended adjuvant therapy with neratinib should also be considered, especially in patients with HR+ disease and residual disease. Patients with HR+/HER2+ IBC should receive 10 years of adjuvant hormonal therapy. Despite significant improved in the neoadjuvant and adjuvant systemic therapy over the past decade, preventing central nervous system recurrence remains an unmet need. Clinical trials evaluating novel anti-HER2 agents, including tucatinib and T-DXd, immune checkpoint inhibitor in combination with T-DM1, and addition of CDK4/6 inhibitor to adjuvant endocrine therapy are underway to improve survival outcomes in the adjuvant therapy setting.

\section{Acknowledgments}

The authors would like to thank Ann Sutton, scientific editor, Research Medical Library, for editing this article.

Funding: None.

\section{Footnote}

Provenance and Peer Review: This article was commissioned by the Guest Editors (Naoto Ueno and Angela Alexander) for the series "Inflammatory Breast Cancer" published in Chinese Clinical Oncology. The article has undergone external peer review.

Reporting Checklist: The authors have completed the Narrative Review reporting checklist. Available at https:// dx.doi.org/10.21037/cco-21-122

Conflicts of Interest: Both authors have completed the ICMJE uniform disclosure form (available at https://dx.doi. org/10.21037/cco-21-122). The series "Inflammatory Breast Cancer" was commissioned by the editorial office without any funding or sponsorship. The authors have no other conflicts of interest to declare.

Ethical Statement: The authors are accountable for all aspects of the work in ensuring that questions related to the accuracy or integrity of any part of the work are appropriately investigated and resolved.

Open Access Statement: This is an Open Access article distributed in accordance with the Creative Commons Attribution-NonCommercial-NoDerivs 4.0 International License (CC BY-NC-ND 4.0), which permits the non- commercial replication and distribution of the article with the strict proviso that no changes or edits are made and the original work is properly cited (including links to both the formal publication through the relevant DOI and the license). See: https://creativecommons.org/licenses/by-nc-nd/4.0/.

\section{References}

1. Zell JA, Tsang WY, Taylor TH, et al. Prognostic impact of human epidermal growth factor-like receptor 2 and hormone receptor status in inflammatory breast cancer (IBC): analysis of 2,014 IBC patient cases from the California Cancer Registry. Breast Cancer Res 2009;11:R9.

2. Lim B, Woodward WA, Wang X, et al. Inflammatory breast cancer biology: the tumour microenvironment is key. Nat Rev Cancer 2018;18:485-99.

3. Chainitikun S, Saleem S, Lim B, et al. Update on systemic treatment for newly diagnosed inflammatory breast cancer. J Adv Res 2020;29:1-12.

4. Gianni L, Pienkowski T, Im YH, et al. 5-year analysis of neoadjuvant pertuzumab and trastuzumab in patients with locally advanced, inflammatory, or early-stage HER2-positive breast cancer (NeoSphere): a multicentre, open-label, phase 2 randomised trial. Lancet Oncol 2016;17:791-800.

5. Schneeweiss A, Chia S, Hickish T, et al. Long-term efficacy analysis of the randomised, phase II TRYPHAENA cardiac safety study: Evaluating pertuzumab and trastuzumab plus standard neoadjuvant anthracycline-containing and anthracycline-free chemotherapy regimens in patients with HER2-positive early breast cancer. Eur J Cancer 2018;89:27-35.

6. Swain SM, Ewer MS, Viale G, et al. Pertuzumab, trastuzumab, and standard anthracycline- and taxane-based chemotherapy for the neoadjuvant treatment of patients with HER2-positive localized breast cancer (BERENICE): a phase II, open-label, multicenter, multinational cardiac safety study. Ann Oncol 2018;29:646-53.

7. van Ramshorst MS, van der Voort A, van Werkhoven ED, et al. Neoadjuvant chemotherapy with or without anthracyclines in the presence of dual HER2 blockade for HER2-positive breast cancer (TRAIN-2): a multicentre, open-label, randomised, phase 3 trial. Lancet Oncol 2018;19:1630-40.

8. van der Voort A, van Ramshorst MS, van Werkhoven ED, et al. Three-Year Follow-up of Neoadjuvant Chemotherapy With or Without Anthracyclines in the Presence of Dual ERBB2 Blockade in Patients With 
ERBB2-Positive Breast Cancer: A Secondary Analysis of the TRAIN-2 Randomized, Phase 3 Trial. JAMA Oncol 2021;7:978-84.

9. Rosenstock AS, Niu J, Giordano SH, et al. Acute myeloid leukemia and myelodysplastic syndrome after adjuvant chemotherapy: A population-based study among older breast cancer patients. Cancer 2018;124:899-906.

10. von Minckwitz G, Untch M, Blohmer JU, et al. Definition and impact of pathologic complete response on prognosis after neoadjuvant chemotherapy in various intrinsic breast cancer subtypes. J Clin Oncol 2012;30:1796-804.

11. van Uden DJP, van Maaren MC, Bult P, et al. Pathologic complete response and overall survival in breast cancer subtypes in stage III inflammatory breast cancer. Breast Cancer Res Treat 2019;176:217-26.

12. Nakhlis F, Regan MM, Warren LE, et al. The Impact of Residual Disease After Preoperative Systemic Therapy on Clinical Outcomes in Patients with Inflammatory Breast Cancer. Ann Surg Oncol 2017;24:2563-9.

13. Diéras V, Miles D, Verma S, et al. Trastuzumab emtansine versus capecitabine plus lapatinib in patients with previously treated HER2-positive advanced breast cancer (EMILIA): a descriptive analysis of final overall survival results from a randomised, open-label, phase 3 trial. Lancet Oncol 2017;18:732-42.

14. Krop IE, Kim SB, Martin AG, et al. Trastuzumab emtansine versus treatment of physician's choice in patients with previously treated HER2-positive metastatic breast cancer (TH3RESA): final overall survival results from a randomised phase 3 study. Lancet Oncol 2017;18:743-54.

15. von Minckwitz G, Huang CS, Mano MS, et al. Trastuzumab Emtansine for Residual Invasive HER2Positive Breast Cancer. N Engl J Med 2019;380:617-28.

16. Mamounas EP, Untch M, Mano MS, et al. Adjuvant T-DM1 versus trastuzumab in patients with residual invasive disease after neoadjuvant therapy for HER2positive breast cancer: subgroup analyses from KATHERINE. Ann Oncol 2021;32:1005-14.

17. Conte P, Schneeweiss A, Loibl S, et al. Patient-reported outcomes from KATHERINE: A phase 3 study of adjuvant trastuzumab emtansine versus trastuzumab in patients with residual invasive disease after neoadjuvant therapy for human epidermal growth factor receptor 2-positive breast cancer. Cancer 2020;126:3132-9.

18. Denkert C, Lambertini C, Fasching PA, et al. Biomarker data from KATHERINE: A phase III study of adjuvant trastuzumab emtansine (T-DM1) versus trastuzumab $(\mathrm{H})$ in patients with residual invasive disease after neoadjuvant therapy for HER2-positive breast cancer. J Clin Oncol 2020;38:502.

19. Martin M, Holmes FA, Ejlertsen B, et al. Neratinib after trastuzumab-based adjuvant therapy in HER2-positive breast cancer (ExteNET): 5-year analysis of a randomised, double-blind, placebo-controlled, phase 3 trial. Lancet Oncol 2017;18:1688-700.

20. Chan A, Moy B, Mansi J, et al. Final Efficacy Results of Neratinib in HER2-positive Hormone Receptor-positive Early-stage Breast Cancer From the Phase III ExteNET Trial. Clin Breast Cancer 2021;21:80-91.e7.

21. Sudhan DR, Schwarz LJ, Guerrero-Zotano A, et al. Extended Adjuvant Therapy with Neratinib Plus Fulvestrant Blocks ER/HER2 Crosstalk and Maintains Complete Responses of ER+/HER2 + Breast Cancers: Implications to the ExteNET Trial. Clin Cancer Res 2019;25:771-83.

22. Barcenas CH, Hurvitz SA, Di Palma JA, et al. Improved tolerability of neratinib in patients with HER2-positive early-stage breast cancer: the CONTROL trial. Ann Oncol 2020;31:1223-30.

23. Gonzalez-Angulo AM, McGuire SE, Buchholz TA, et al. Factors predictive of distant metastases in patients with breast cancer who have a pathologic complete response after neoadjuvant chemotherapy. J Clin Oncol 2005;23:7098-104.

24. Fei F, Messina C, Slaets L, et al. Tumour size is the only predictive factor of distant recurrence after pathological complete response to neoadjuvant chemotherapy in patients with large operable or locally advanced breast cancers: a sub-study of EORTC 10994/BIG 1-00 phase III trial. Eur J Cancer 2015;51:301-9.

25. Piccart M, Procter M, Fumagalli D, et al. Adjuvant Pertuzumab and Trastuzumab in Early HER2-Positive Breast Cancer in the APHINITY Trial: 6 Years' FollowUp. J Clin Oncol 2021;39:1448-57.

26. Modi S, Saura C, Yamashita T, et al. Trastuzumab Deruxtecan in Previously Treated HER2-Positive Breast Cancer. N Engl J Med 2020;382:610-21.

27. Cortés J, Kim SB, Chung WP, et al. Trastuzumab deruxtecan (T-DXd) vs trastuzumab emtansine (T-DM1) in patients (Pts) with HER2 + metastatic breast cancer (mBC): Results of the randomized phase III DESTINYBreast03 study. An Oncol 2021;32:S1287-8.

28. Murthy RK, Loi S, Okines A, et al. Tucatinib, Trastuzumab, and Capecitabine for HER2-Positive Metastatic Breast Cancer. N Engl J Med 2020;382:597-609.

29. Lin NU, Borges V, Anders C, et al. Intracranial Efficacy 
and Survival With Tucatinib Plus Trastuzumab and Capecitabine for Previously Treated HER2-Positive Breast Cancer With Brain Metastases in the HER2CLIMB Trial. J Clin Oncol 2020;38:2610-9.

30. Schmid P, Rugo HS, Adams S, et al. Atezolizumab plus nab-paclitaxel as first-line treatment for unresectable, locally advanced or metastatic triple-negative breast cancer (IMpassion130): updated efficacy results from a randomised, double-blind, placebo-controlled, phase 3 trial. Lancet Oncol 2020;21:44-59.

31. Cortes J, Cescon DW, Rugo HS, et al. Pembrolizumab plus chemotherapy versus placebo plus chemotherapy for previously untreated locally recurrent inoperable or metastatic triple-negative breast cancer (KEYNOTE-355): a randomised, placebo-controlled, double-blind, phase 3 clinical trial. Lancet 2020;396:1817-28.

32. Schmid P, Cortes J, Pusztai L, et al. Pembrolizumab for Early Triple-Negative Breast Cancer. N Engl J Med 2020;382:810-21.

33. Loi S, Giobbie-Hurder A, Gombos A, et al. Pembrolizumab plus trastuzumab in trastuzumab-resistant, advanced, HER2-positive breast cancer (PANACEA): a single-arm, multicentre, phase 1b-2 trial. Lancet Oncol 2019;20:371-82.

34. Emens LA, Esteva FJ, Beresford M, et al. Trastuzumab emtansine plus atezolizumab versus trastuzumab emtansine plus placebo in previously treated, HER2positive advanced breast cancer (KATE2): a phase 2, multicentre, randomised, double-blind trial. Lancet Oncol 2020;21:1283-95.

35. Harbeck N, Rastogi P, Martin M, et al. Adjuvant abemaciclib combined with endocrine therapy for high-risk early breast cancer: updated efficacy and $\mathrm{Ki}$ 67 analysis from the monarchE study. Ann Oncol

Cite this article as: Zakon DB, Valero V. What is the best treatment recommendation for HER2 + IBC with residual disease?-a narrative review. Chin Clin Oncol 2021;10(6):59. doi: $10.21037 /$ cco-21-122
2021;32:1571-81.

36. Patnaik A, Rosen LS, Tolaney SM, et al. Efficacy and Safety of Abemaciclib, an Inhibitor of CDK4 and CDK6, for Patients with Breast Cancer, Non-Small Cell Lung Cancer, and Other Solid Tumors. Cancer Discov 2016;6:740-53.

37. Tolaney SM, Wardley AM, Zambelli S, et al. Abemaciclib plus trastuzumab with or without fulvestrant versus trastuzumab plus standard-of-care chemotherapy in women with hormone receptorpositive, HER2-positive advanced breast cancer (monarcHER): a randomised, open-label, phase 2 trial. Lancet Oncol 2020;21:763-75.

38. Francis PA, Pagani O, Fleming GF, et al. Tailoring Adjuvant Endocrine Therapy for Premenopausal Breast Cancer. N Engl J Med 2018;379:122-37.

39. Goss PE, Ingle JN, Martino S, et al. A randomized trial of letrozole in postmenopausal women after five years of tamoxifen therapy for early-stage breast cancer. N Engl J Med 2003;349:1793-802.

40. Davies C, Pan H, Godwin J, et al. Long-term effects of continuing adjuvant tamoxifen to 10 years versus stopping at 5 years after diagnosis of oestrogen receptorpositive breast cancer: ATLAS, a randomised trial. Lancet 2013;381:805-16.

41. Early Breast Cancer Trialists' Collaborative Group (EBCTCG). Adjuvant bisphosphonate treatment in early breast cancer: meta-analyses of individual patient data from randomised trials. Lancet 2015;386:1353-61.

42. Dhesy-Thind S, Fletcher GG, Blanchette PS, et al. Use of Adjuvant Bisphosphonates and Other Bone-Modifying Agents in Breast Cancer: A Cancer Care Ontario and American Society of Clinical Oncology Clinical Practice Guideline. J Clin Oncol 2017;35:2062-81. 